\title{
Methods for Full Utilization of the Bandwidth of Fiber Optical Parametric Amplifiers and Wavelength Converters
}

\author{
Georgios Kalogerakis, Student Member, IEEE, Michel E. Marhic, Senior Member, IEEE, \\ Katsumi Uesaka, Member, IEEE, Katsuhiro Shimizu, Member, IEEE, Member, OSA, \\ Kenneth Kin-Yip Wong, Student Member, IEEE, and Leonid G. Kazovsky, Fellow Member, IEEE, Fellow, OSA
}

\begin{abstract}
In fiber optical parametric amplifiers (OPAs), idlers are generated during the amplification process. For very wide and dense signal input spectra, idlers may overlap with signals, thereby interfering with proper operation as an amplifier. In this paper, filter-based methods to fully utilize the bandwidth of OPAs and wavelength converters in the presence of very broad signal spectra are investigated. In the basic filter setup, two parallel OPAs and two filters are used; alternatively, one can use a single OPA bidirectionally and a single filter. An interleaver-based arrangement, separating signals from idlers at the system output, with a crosstalk of below $-20 \mathrm{~dB}$ and subdecibel bit-error-rate penalty, is experimentally demonstrated.
\end{abstract}

Index Terms-Interleaving filters, nonlinear fibers, optical amplifier, optical parametric amplifier (OPA), wavelength conversion.

\section{INTRODUCTION}

$\mathbf{F}$ IBER-BASED optical parametric amplifiers (OPAs) [1] appear to be a promising technology for amplification and band conversion in future wavelength-division multiplexing (WDM) systems and next-generation optical networks due to their large and flexible gain bandwidths [2]-[5]. Bandwidths of several hundred nanometers [2]-[6] and internal continuouswave $(\mathrm{CW})$ gains of the order of 50-60 dB have recently been reported [3], [7]. The development of novel low-dispersion highly nonlinear fibers (HNLFs) [8], [9] and high-power pumps could further enhance the performance of parametric amplifiers to a level such that they could become practical for optical communication systems.

The presence of an idler at the output for each signal can also be used for making very wideband wavelength converters

Manuscript received January 6, 2006; revised July 6, 2006. This work was supported in part by the National Science Foundation under Grants ANI0123441 and ECS-9821026.

G. Kalogerakis, M. E. Marhic, and L. G. Kazovsky are with the Department of Electrical Engineering, Stanford University, Stanford, CA 94305 USA (e-mail: gkalog@stanford.edu).

K. Uesaka is with Sumitomo Electric Industries Ltd., Yokohama 244-0844, Japan.

K. Shimizu is with Information Technology R\&D Center, Mitsubishi Electric Corporation, Kamakura 247-8501, Japan.

K. K.-Y. Wong is with the Department of Electrical and Electronic Engineering, University of Hong Kong, Pokfulam, Hong Kong.

Color versions of Figs. 3, 4, 7, and 8(b) are available online at http://ieeexplore.ieee.org.

Digital Object Identifier 10.1109/JLT.2006.882248
[10], [11]. However, this property of fiber OPAs based on fourwave mixing (FWM) is detrimental when we try to use the full OPA bandwidth. If we suppose that a densely populated WDM spectrum at the input of an OPA covers its entire gain bandwidth, then at the output, the signal and idler spectra overlap completely. Therefore, only half of the available gain bandwidth is usable for input signals in a conventional singleOPA configuration. In this paper, we investigate possible means for modifying the basic OPA configuration in order to use input signals filling the entire gain bandwidth. This paper is organized as follows: In Section II, we discuss the use of filters to partition the input spectrum and to separate signals from idlers at the output. In Section III, we present the experimental results. In particular, we demonstrate the implementation of an interleaver-based scheme with a crosstalk of below $-18 \mathrm{~dB}$ and subdecibel bit-error-rate (BER) penalty. We discuss various aspects of this scheme in Section IV and conclude in Section V.

\section{PRINCIPLE OF OPERATION}

\section{A. Filter-Based Approach}

To avoid the overlap of signals and idlers at the output of an OPA, which amplifies a dense WDM (DWDM) spectrum filling its gain spectrum, it is clear that one cannot use a single OPA. One must use at least two OPAs, each amplifying only a subset of the original signal wavelengths. One must then partition the input wavelengths in such a way that for each OPA, the idlers will not overlap with the signals. If this can be done, one can then in principle use other optical filters to separate the signals from the idlers after each OPA. Then, by using additional filters, one can combine the amplified signals (idlers) of both OPAs if one wants to use the system as an amplifier (wavelength converter).

Here, we are going to introduce a procedure for specifying the transmittance spectra of filters that are suitable for partitioning the set of input signals. We will also show that the filters required to recombine the signals and idlers at the output can actually be of the same type as the input filter. We will consider several systems that can be designed based on such filters and OPAs. We will then discuss the limitations imposed by the physical realizability of the filters. 


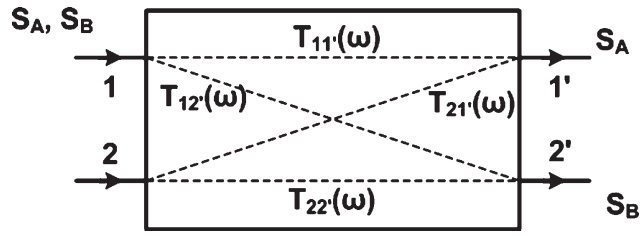

Fig. 1. Two-port lossless optical filter.

We begin by investigating the first filter, which is necessary to partition the signals. We assume that the number $N_{c}$ of carriers is a multiple of 2, i.e., $N_{c}=2 N$, where $N$ is an integer. To simplify the discussion, we initially consider two-input two-output filters with binary transmittance functions, i.e., with possible values of 0 or 1 in several bands. We also assume that the transitions between passbands and stopbands occur halfway between two optical carriers. We let $\omega$ denote the angular frequency, and we assume that $\omega=0$ is in the middle of the WDM spectrum, i.e., halfway between carriers $N$ and $N+1$.

Consider then the two-port filter in Fig. 1. The $2 N$ carriers are incident at port 1 . We first construct the desired transmittance function $T_{11^{\prime}}(\omega)$ between ports 1 and $1^{\prime}$ as follows.

1) Consider the $N$ signals with carriers such that $\omega<0$. We partition them into two arbitrary groups. We choose $T_{11^{\prime}}=1$ for one group and $T_{11^{\prime}}=0$ for the other group. This completely specifies $T_{11^{\prime}}(\omega)$ for $\omega<0$.

2) To construct $T_{11^{\prime}}(\omega)$ for $\omega>0$, we impose the condition $T_{11^{\prime}}(\omega)=1-T_{11^{\prime}}(-\omega)$. Rewritten as $T_{11^{\prime}}(\omega)-1 / 2=$ $-\left[T_{11^{\prime}}(-\omega)-1 / 2\right], T_{11^{\prime}}(\omega)$ exhibits an odd symmetry about the point $(0,1 / 2)$. This is a requirement that is also encountered with filters for other applications, such as single-sideband communication [12].

As a result of this construction, $T_{11^{\prime}}(\omega)$ will correspond to the transmission of exactly half the number of input signals (i.e., $N$ ). Fig. 2 shows an example of $T_{11^{\prime}}(\omega)$ for $N_{c}=12$, which is constructed by arbitrarily partitioning the six carriers with $\omega<0$.

If we also assume that the filter is lossless, energy conservation dictates that we must have $T_{12^{\prime}}(\omega)=1-T_{11^{\prime}}(\omega)$. Thus, those original signals that do not come out of port $1^{\prime}$ must come out of port $2^{\prime}$, i.e., the signal spectra at $1^{\prime}$ and $2^{\prime}$ are complementary, forming a partition of the original signal spectrum into two groups of $N$ signals. We denote the group of signals emerging from the upper (lower) output of the filter in Fig. 1 by $S_{A}\left(S_{B}\right)$.

Consider now Fig. 3(a). If we feed $S_{A}$ into an OPA (OPA1) with a center frequency at $\omega=0, N$ idlers are generated, which are symmetric of the signals with respect to $\omega=0$; we denote this group by $I_{A}$ (the frequencies in $I_{A}$ are the same as in $S_{B}$ ). By construction of $T_{11^{\prime}}(\omega)$ and thus of $S_{A}$, all the idlers of $I_{A}$ fall into gaps between the signals of $S_{A}$. Hence, at the output of OPA1, we have $N$ signals and $N$ idlers, forming a group of $2 N$ evenly spaced carriers.

If we now pass $S_{B}$ through a second OPA (OPA2), which is identical to OPA1, we again obtain at its output a set of $N$ signals $S_{B}$ and $N$ idlers, which we denote by $I_{B}$ (the frequencies in $I_{B}$ are the same as in $S_{A}$ ). So again, we have a set of $2 N$ evenly spaced carriers.

Let us now assume that we want to eventually gather all signals in one fiber and all idlers in another one. To do so, we will use one or more additional filters similar to the first one and the following properties: 1) $T_{21^{\prime}}(\omega)=T_{12^{\prime}}(\omega)$, and $\left.T_{22^{\prime}}(\omega)=T_{11^{\prime}}(\omega) .2\right)$ If we use the same filter backward, the transmittance of any input-output path is the same in both directions.

There are several ways to accomplish the recombination of signals, which involves tradeoffs. We describe the main possibilities in the succeeding subsections.

1) Two-Filter Two-OPA System: This arrangement is shown in Fig. 3(a). The particular nature of the OPA output spectra and of the filter suggests that we can actually accomplish the task by using only a second filter, which is identical to the first one. We assume for simplicity that the OPAs have constant unit gain for all signals and idlers (actually, it will probably be a large gain, which is slightly different for signals and idlers, but this is not important here). Then, the total transmittance function from 1 to $3^{\prime}$ is

$$
\begin{aligned}
T_{11^{\prime}}(\omega) T_{33^{\prime}}(\omega)+T_{12^{\prime}}(\omega) T_{43^{\prime}}(\omega) & =\left[T_{11^{\prime}}(\omega)\right]^{2}+\left[T_{12^{\prime}}(\omega)\right]^{2} \\
& =T_{11^{\prime}}(\omega)+T_{12^{\prime}}(\omega) \\
& =1 \quad \forall \omega .
\end{aligned}
$$

This shows that all the signals indeed appear at a single output $\left(3^{\prime}\right)$ of the whole system, as desired. Tracing the idlers through the system is a bit more complicated, as they cannot be directly handled by transmittance functions because of the frequency inversion. With care, however, we can use the notion of "equivalent" transmittance functions for the idlers. For example, we can say that the idler spectrum after OPA1 (OPA2) is obtained from the entire signal input spectrum ("not" filtered by the first filter), multiplied by $T_{12^{\prime}}(\omega)\left[T_{11^{\prime}}(\omega)\right]$. Then, the idler spectrum at output $4^{\prime}$ is given by

$$
\begin{aligned}
& T_{12^{\prime}}(\omega) T_{34^{\prime}}(\omega)+T_{11^{\prime}}(\omega) T_{44^{\prime}}(\omega) \\
& \quad=\left[T_{12^{\prime}}(\omega)\right]^{2}+\left[T_{11^{\prime}}(\omega)\right]^{2}=T_{12^{\prime}}(\omega)+T_{11^{\prime}}(\omega)=1 .
\end{aligned}
$$

This shows that all the idlers indeed appear at a single output $\left(4^{\prime}\right)$ of the whole system.

Altogether then, we verify that this system, using only two identical filters, is suitable for separating the amplified signals from the idlers generated within the OPAs. Such a system can be used as an amplifier, a wavelength converter, or both.

2) Five-Filter Two-OPA System: Another approach is shown in Fig. 3(b). Filters F1-F5 are all identical and designed as in the preceding section. We use F2 to separate the signals from the idlers after OPA1 and F3 to do the same after OPA2. $S_{A}$ and $S_{B}$, respectively, appear at their upper outputs, while $I_{A}$ and $I_{B}$ appear at their lower outputs. Then, F4 combines the two groups of signals, and F5 combines the two groups of idlers. It is clear that $S_{A}$ and $S_{B}$ appear at the upper output of F4, while $I_{A}$ and $I_{B}$ appear at the lower output of F5; this provides the desired result. While this approach will accomplish the task, it could prove expensive due to the use 


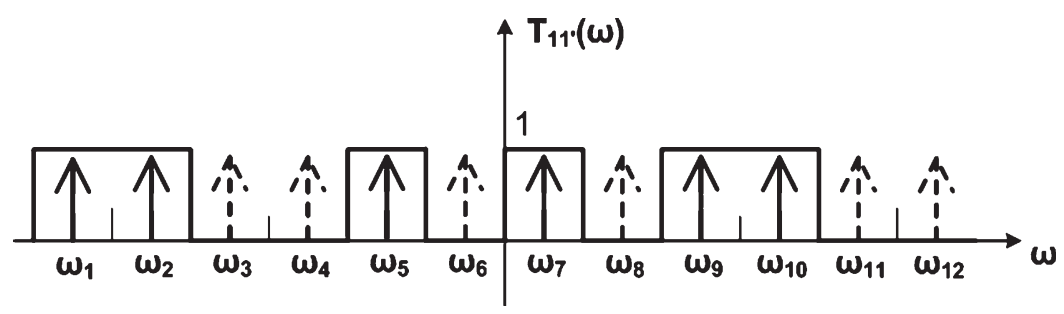

Fig. 2. Binary transmittance versus frequency, which is suitable for working with an OPA. The solid (dashed) arrows represent the carriers that are (not) transmitted from 1 to $1^{\prime}$. These sets are denoted as $S_{A}$ and $S_{B}$.

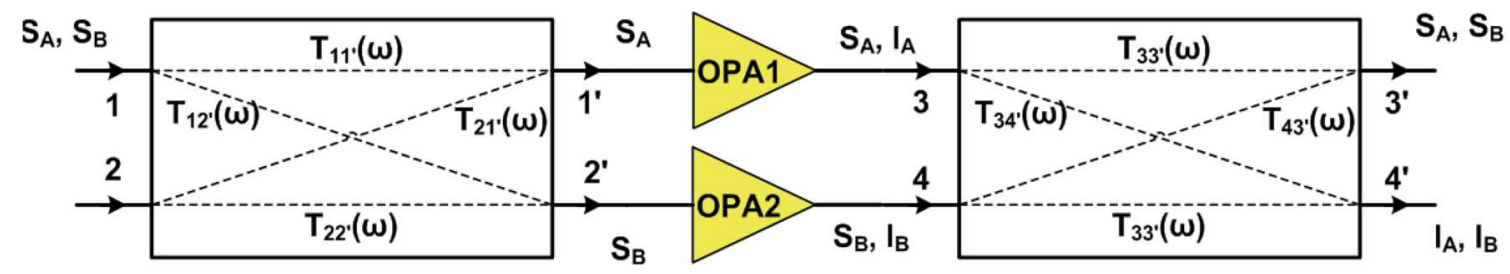

(a)

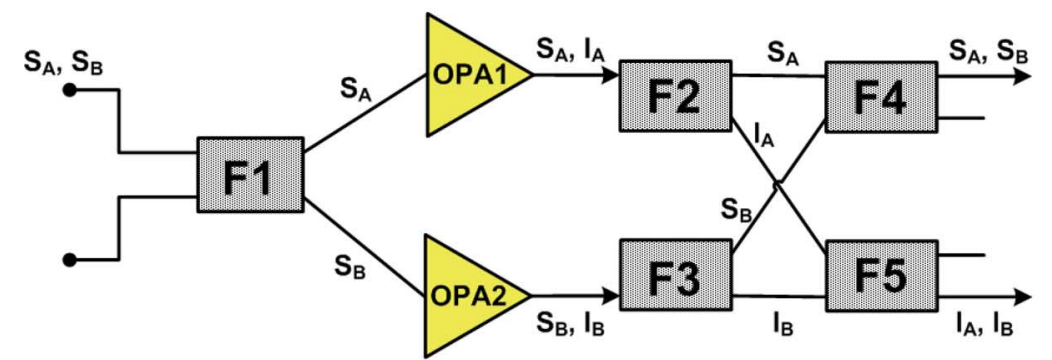

(b)

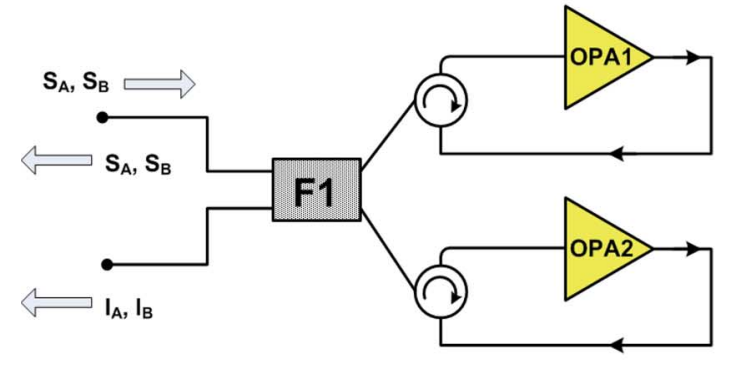

(c)

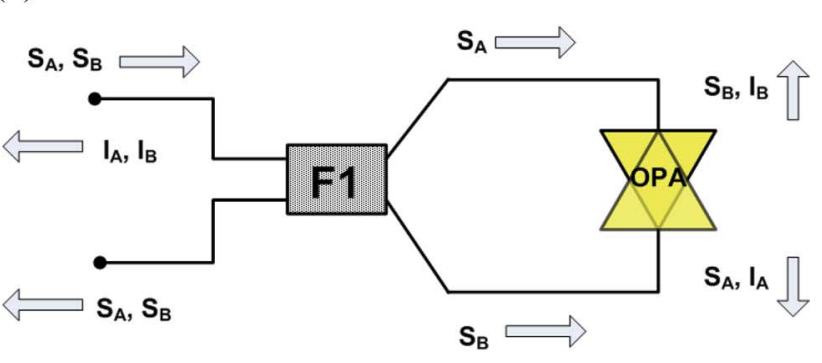

(d)

Fig. 3. (a) Two-filter two-OPA. (b) Five-filter two-OPA. (c) One-filter two-OPA. (d) One-filter one-bidirectional-OPA systems.

of five separate filters. On the other hand, we note that in many applications, one is interested in either the output signals or the idlers but not in both; in that case, either F4 or F5 is unnecessary, which leads to some simplification. An important reason for mentioning this five- or four-filter approach is that it presents a sizeable advantage when it comes to crosstalk between signals and idlers. Because the version in Fig. 3(b) has open output ports, unwanted crosstalk will exit through these ports, instead of being superimposed on the desired frequency components, which can occur in Fig. 3(a), where there are no unused output ports. This is essentially the same idea that is used for improving the extinction ratio in optical switches by designing so-called dilated switches [13].

3) One-Filter Two-OPA System: Because the filter operates in essentially the same manner forward and backward, we can use a single filter in both directions, as shown in Fig. 3(c). Circulators are needed to extract signals and idlers from the OPAs and return them to the filter. In this case, a circulator (not shown) must be used to extract the output signals as they exit the system, traveling backward in the input fiber.

4) One-Filter One-OPA Bidirectional System: Because an OPA amplifies only in the direction of the pump, we can actually use a single nonlinear fiber with two counterpropagating pumps to implement OPA1 and OPA2 in the same fiber, each amplifying in a different direction. We can then use this feature, as shown in Fig. 3(d), to use a single bidirectional filter. Here, a circulator must be used to extract the output idlers from the input fiber. This is probably the simplest system that can be designed to perform the desired functions. An additional advantage of this approach is that since both 
OPAs use exactly the same fiber, they will have the same gain spectra if the same pump power is used in both directions (in addition, loss should be negligible, and the states of polarization of the waves should be carefully controlled) [14]. This can be advantageous for avoiding ripple in the output spectra, which would result from poorly matched gain spectra in two different OPAs.

One other advantage of the simplest configurations 3 and 4 with respect to the more complicated ones (configurations 1 and 2) is that since the input and output filters are essentially the same filter, their passbands need not to be mutually aligned. Furthermore, configuration 4 is the most cost effective in terms of the number of active components used because the bidirectional OPA requires a single HNLF, as will be evident in Section III-A. On the other hand, one serious disadvantage of these simpler architectures is that signals experience larger insertion losses since they transverse through a number of necessary circulators. Therefore, more pump power is needed in order to maintain the same output signal power. This may not be acceptable for applications where power consumption is critical.

\section{B. Considerations for Physically Realizable Filters}

In the preceding section, we presented the principle of our method based on idealized filters with binary transmittance functions. Physically realizable filters, however, only approximate these ideal functions. They actually have ripple in the passband, finite extinction in the stop band, and finite slope in the transition region from passband to stopband. We now consider physically realizable filters, with realistic transmittance functions, and investigate the limitations brought about by their characteristics.

Realizable Partitions of the Input Spectrum: While it is in principle possible to design optical filters with arbitrary complex transfer functions, their implementation might require an infinite number of elements (layers in a multilayer dielectric mirror or stages in a lattice filter), and this would prevent their fabrication. The state of the art for multilayer dielectric mirror limits the number of layers to about 1000 ; for lattice filters, the maximum number of stages is a few tens. These finite numbers lead to the aforementioned defects when compared with ideal binary filters. A related consideration is that it is easier to design and fabricate periodic filters than nonperiodic ones. For example, for lattice filters, periodicity can be obtained by having equal delays in all the stages [15]. In view of this, we now restrict our discussion to periodic filters, which greatly reduces the number of partitions of the input signal that can be considered. Periodic filters are also required when dealing with standard WDM spectra, with the carriers regularly spaced on a frequency grid.

The requirement that the periodic input filter must partition the $2 N$ input signals into two groups of $N$ immediately imposes that one passband and one stopband must contain the same number of signals $N_{b}$. The detailed shapes of the passband and the stopband spectra are very important, and they can be chosen for other applications by specifying parameters such as passband ripple, sharpness of the transition between passband and stopband, symmetry within passband and stopband, and contrast between passband and stopband.

The sharpness of the transition between passband and stopband is important for our application. If we consider a lattice filter with $N_{l}$ stages and a time delay per stage $\Delta \tau$, the filter period in the $\omega$ domain is $\Omega_{f}=1 / \Delta \tau$, and the width $\delta \omega$ of the transition is proportional to $\Omega_{f} / N_{l}=1 /\left(N_{l} \Delta \tau\right)$. Since $N_{l}$ has a maximum practical value, the only way to have a sharp transition is to increase $\Delta \tau$, which in turn reduces $\Omega_{f}$. As a result, the filters with the sharpest transitions will be those with the narrowest passbands, i.e., those with $N_{b}=1$. These are interleavers that partition the input signals into even and odd groups. Compared to the spectrum of one signal, they will have the sharpest transitions and, thus, will look more square than filters with wider passbands. In fact, if we now go to the opposite limit, i.e., if we let $N_{b}=N$, we will have a filter that will partition the signals into positive and negative frequencies. However, the transition between passband and stopband will be very wide, and for a large $N$, it could well extend over several channels. This would be unacceptable in systems where it is desired to have useful channels in the middle of the signal spectrum. In such situations, the opposite limit, i.e., that of an interleaver with one signal per passband, becomes attractive, as no channels would be lost near the center of the WDM spectrum.

We thus see that there are tradeoffs between these two extreme cases. The interleaver can in principle utilize all the channels of a DWDM spectrum, but each channel will be affected by the shape of the passband, particularly in multistage systems. On the other hand, a high-pass-low-pass band filter will present a gap in the middle, but the individual channel spectra will not be affected much by the passband ripple, even in cascaded systems.

\section{EXPERIMENTS AND RESULTS}

\section{A. Principle Demonstration}

We have performed experiments to demonstrate the feasibility of implementing simple versions of the proposed architectures with commonly available components [16]. The first experimental configuration implements the principle in Fig. 3(c) and is shown in Fig. 4(a). A number of signals are fed into the input of the interleaver. Odd and even signals emerge from either port of the interleaver and are amplified in the corresponding OPA. The same pump wavelength is used for both OPAs. As explained in Section II, the choice of pump wavelength is such that the idler wavelengths fall precisely where the signals have been removed by the interleaver. The outputs of the OPAs are then fed back into the same interleaver, which combines them in such a way that all the original signals emerge at its output. The input and output ports in this case are identical as far as the proposed architecture is concerned. A circulator is used to extract the output spectrum. With a four-port interleaver, all the idlers would emerge at the other (complementary) output; thus, both the original and a spectrally inverted version of the input WDM spectrum would be available. 




(a)

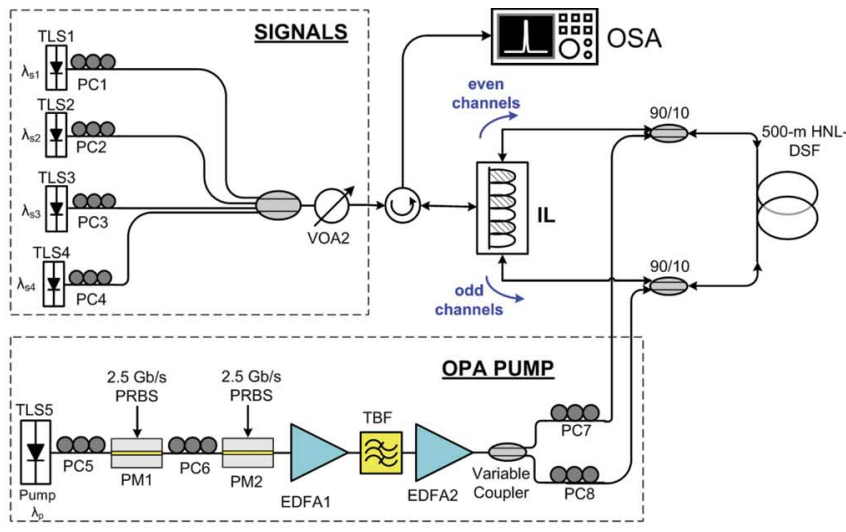

(b)

Fig. 4. (a) Experimental setup for full-bandwidth OPA. (b) Corresponding bidirectional setup (tunable laser source, TLS; polarization controller, PC; phase modulator, PM; interleaver, IL; optical spectrum analyzer, OSA).

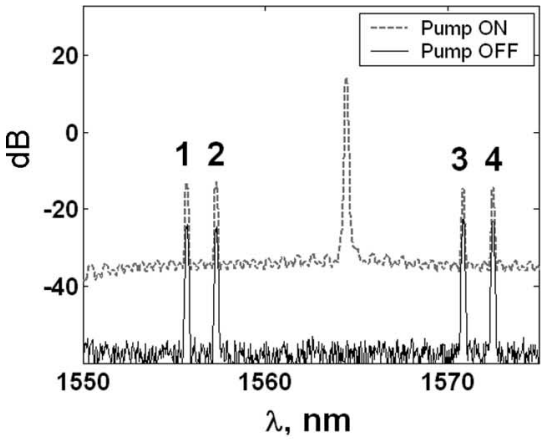

(a)



(b)

Fig. 5. (a) Output spectra for full-bandwidth OPA (with interleaver), when the pump is either ON or OFF. (b) Output spectra when the OPA outputs are combined by a coupler $\left(1,2,3\right.$, 4 : signals; $1^{\prime}, 2^{\prime}, 3^{\prime}, 4^{\prime}$ : idlers).

We used an external-cavity tunable laser as the pump source. Two phase modulators that are driven at $2.5 \mathrm{GHz}$ were used to broaden the pump spectrum in order to suppress stimulated Brillouin scattering (SBS). The resulting pump was then amplified by a cascade of two erbium-doped fiber amplifiers (EDFAs): EDFA1, which acts as a preamplifier, and EDFA2, which serves as a booster amplifier to bring the pump power to the desired level. A bandpass filter was inserted between these two EDFAs to suppress amplified spontaneous emission (ASE) noise. A variable coupler then split the pump power, and each part was fed into a different spool of HNLF through 90/10 couplers (in a practical amplifier, dichroic couplers should be used to avoid the 10-dB insertion loss of the signal in each branch). The use of the variable coupler was necessary because the two OPAs needed different amounts of pump power due to slightly different fiber lengths, zero-dispersion wavelengths, and conversion efficiencies. The two fibers used as nonlinear media for the OPAs had different zero-dispersion wavelengths $\lambda_{0}$ of 1561.14 and $1564.1 \mathrm{~nm}$. Their dispersion slope was $0.03 \mathrm{ps} / \mathrm{nm}^{2} \mathrm{~km}$, their fourth-order dispersion coefficient was $\left(\beta_{4}\right)-5.810^{-56} \mathrm{~s}^{4} / \mathrm{m}$, their nonlinear coefficient was $11 \mathrm{~km}^{-1} \cdot \mathrm{W}^{-1}$, and the attenuation coefficient was $0.5 \mathrm{~dB} / \mathrm{km}$. The pump wavelength $\lambda_{p}$ was set at $1564.34 \mathrm{~nm}$, which is halfway between two adjacent even and odd channels, as discussed in Section II. With this choice, we could simultaneously optimize the gain spectra of the two OPAs. The pump power at the input of either HNLF was $25 \mathrm{dBm}$. Four tunable laser sources served as signals. They were combined by couplers and fed into the interleaver. The outputs of the interleaver were then amplified by the HNLFs. Idler removal was achieved by passing the amplified signals and the corresponding idlers again through the same interleaver by using circulators, as shown in Fig. 4(a). Polarization controllers were inserted at various locations to align the polarization states of the pump and the signals.

The output ASE spectrum shape was monitored with the Optical Society of America (OSA) and eventually optimized by properly distributing the pump power between the two branches with the variable coupler. The signal wavelengths were set at $1555.71,1557.32,1570.88$, and $1572.49 \mathrm{~nm}$, and the highpower EDFA output power was set at $31.8 \mathrm{dBm}$. Half of the signals corresponded to odd channels, and the other half corresponded to the even ones. Therefore, each OPA branch amplified the same number of channels.

The spectrum at the output of the configuration under these conditions is shown in Fig. 5(a). The spectrum when the pump is OFF is also shown in the same figure. The pump wavelength is such that the idlers of the odd channels (1555.71 and $1570.88 \mathrm{~nm}$ ) fall exactly at positions such that they do not appear at the single output of the interleaver (with a four-port 




Fig. 6. Output spectra for bidirectional full-bandwidth OPA when the pump is either ON or OFF.

interleaver, the idlers would be present at the second output port). The same applies for the even channels (1557.32 and $1572.49 \mathrm{~nm})$. We confirm that the signals are amplified with gain values of $11,11.7,7.9$, and $8.44 \mathrm{~dB}$, respectively, and that no idlers are present at the output. Although the measurements were limited by EDFA2 ASE noise, the extinction ratio, which depends primarily on the quality of the interleaver and corresponds to the idler suppression ratio, is better than $18 \mathrm{~dB}$. It should be noted here that no filter was used at the output of the booster EDFA2 for these measurements. As a result, the ASE noise from the EDFA was not filtered out. The noise floor at the output of the OPA does not correspond to the actual gain profile of the OPA, which is not flat, as expected from a onepump configuration.

In a separate experiment, we connected the outputs of the HNLFs with a 3-dB coupler. The output spectrum was monitored for comparison and is shown in Fig. 5(b). It is similar to that of a conventional OPA. Idlers are present at the output; therefore, it is impossible to utilize the full OPA bandwidth with this latter configuration when a DWDM spectrum is present at the input.

The setup for a full-bandwidth OPA using a single HNLF in a bidirectional configuration is shown in Fig. 4(b). In this implementation, the outputs of the interleaver were connected to the two ends of a single $0.5-\mathrm{km}$ HNLF spool. The HNLF with a zero-dispersion wavelength of $1564.1 \mathrm{~nm}$ was used. Hence, the even and odd signals counterpropagated inside the same OPA fiber. The pump configuration was exactly the same as in the previous experiment, and it was inserted by $90 / 10$ couplers into the fiber. The pump power inserted into the HNLF in either direction was $25 \mathrm{dBm}$, and the pump wavelength was again at $1564.34 \mathrm{~nm}$. The two counterpropagating pumps separately amplified the even and odd signals. As expected, idlers were again rejected by the interleaver. Only the amplified signals could be observed at the output, as shown in Fig. 6. The gain values of the channels were 7.9, 8.5, 10.2, and $12 \mathrm{~dB}$, respectively. This setup has the advantage that the gain medium for both the even and odd signals is exactly the same, which facilitates gain spectrum optimization and results in a larger bandwidth. On the other hand, the bidirectional operation may result in cross-phase modulation (XPM) penalties due to the interaction between counterpropagating pumps [17].

\section{B. Performance Measurements}

The experimental setup in Fig. 7 was used for performance measurements. It differed from the one described in Section III-A in that we split the pump before amplifying it to the desired level using two separate high-power EDFAs. In addition, we used optical filters at the outputs of each booster EDFA to reduce the ASE noise level that enters the highly nonlinear dispersion-shifted fiber (HNL-DSF) [18]. The optical signal-to-noise ratio (OSNR) at the input of each HNL-DSF was more than $55 \mathrm{~dB}$ in order to ensure high-quality signal amplification [19] and minimize the intensity noise transfer from the pump to the signals and idlers [20]. The signal from TLS4 (channel 4) was tuned at $1573.72 \mathrm{~nm}$ and nonreturnto-zero (NRZ) modulated at $10 \mathrm{~Gb} / \mathrm{s}$. Three other signals in the $C$-band (channels 1-3) were placed in adjacent positions of the interleaver passband. Their wavelengths were 1554.91, 1555.34 , and $1555.81 \mathrm{~nm}$, respectively. They were combined and modulated at $10 \mathrm{~Gb} / \mathrm{s}$ using an external Mach-Zehnder modulator (MZM). The three $C$-band channels were combined with the $L$-band one by means of a WDM coupler. The pump wavelength was tuned at $1564.16 \mathrm{~nm}$. The selection of the pump and signal wavelengths was such that the idler corresponding to channel 4 fell exactly at the wavelength of channel 2 and vice versa.

Channels 1, 3, and 4 were routed into HNL-DSF 1 through the interleaver. The powers of those signals at the fiber input were $-22,-18.4$, and $-20.75 \mathrm{dBm}$, respectively. The corresponding pump power at this point was $25.2 \mathrm{dBm}$. The input-signal power was kept low enough to avoid cross-gain modulation effects, which are present in OPAs in the presence of WDM signals [21]. Channel 2 was separated by the interleaver and entered HNL-DSF 2 with an input power of $-18.4 \mathrm{dBm}$, where it was amplified by a $27.9-\mathrm{dBm}$ pump. The ON-OFF gain of channel 2 was about $10 \mathrm{~dB}$. Note that we used different pump powers for the two fibers. Although these fibers come from the same fiber spool, they have slightly different zero-dispersion wavelengths and therefore, slightly different gain profiles if driven by a common-wavelength pump. The amplified signals were routed back into the interleaver, as described in Section III-A. Fig. 8(a) shows the spectrum at the output of the setup. Channel 2 was then further selected with a narrow-band filter, and BER measurements were performed for three scenarios: 1) Only channel 2 was ON. 2) Channels 2 and 4 were ON (the wavelengths of the idlers that corresponded to channels 2 and 4 fell exactly at the wavelengths of channel 4 and 2, respectively). 3) All channels were ON. The BER measurement results are summarized in Fig. 8(b). The penalty at a BER level of $10^{-9}$ is around $0.9 \mathrm{~dB}$. We conclude that as we proceeded from case 1 to case 3 and more channels were turned on, no additional penalty was observed. The low penalty confirms the viability of the proposed architecture. These performance measurements can act as a reference point for the rest of the configurations suggested in Section II-A. In particular, the performance of the bidirectional OPA configuration is expected to be similar or slightly worse due to an additional XPM penalty, which is associated with bidirectional systems [17]. Further analysis on the performance comparison of the various proposed architectures is left for future research work. 


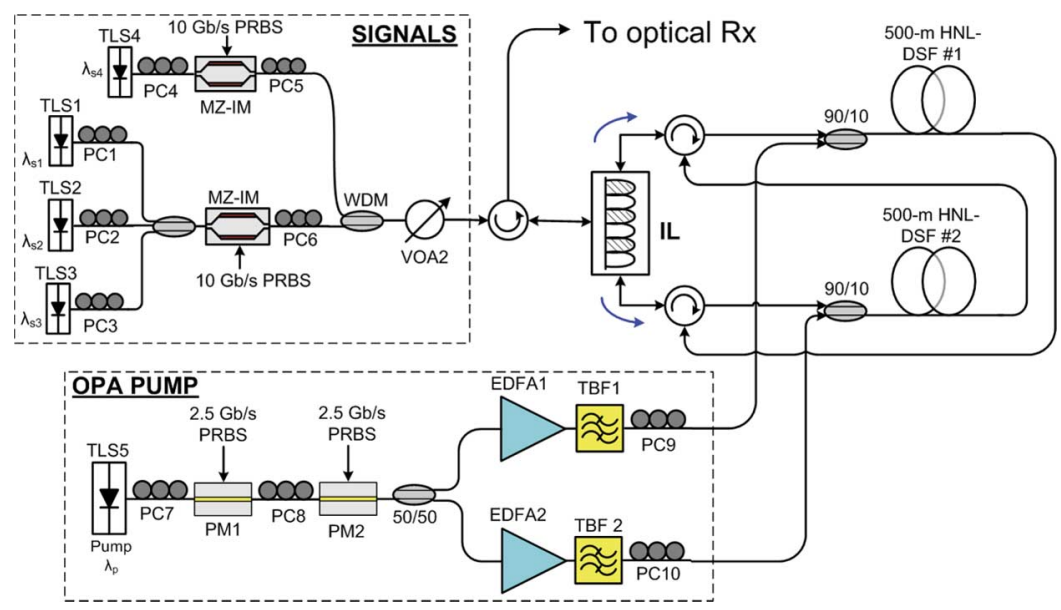

Fig. 7. Experimental setup for performance measurements.

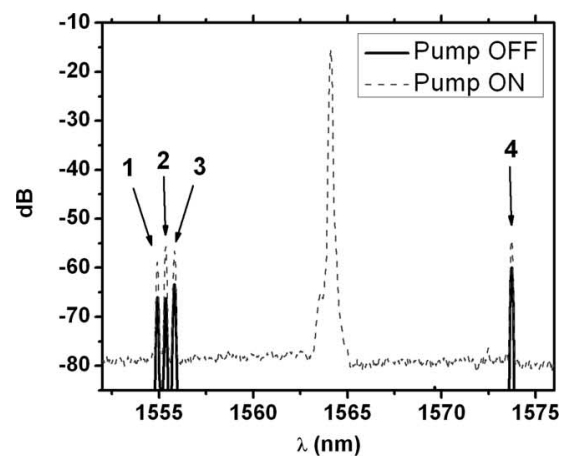

(a)

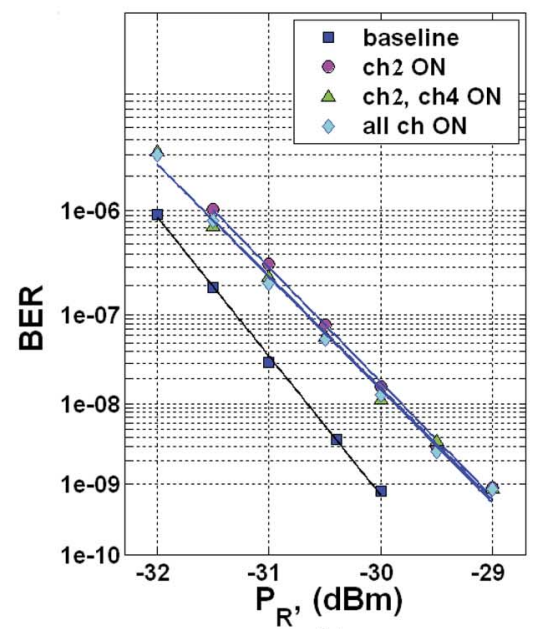

(b)

Fig. 8. (a) Spectra at the output of the performance measurement experimental setup when the pump is either ON or OFF. The resulting idler of channel 4 falls exactly at the wavelength of channel 2 and vice versa. (b) BER plots for three different scenarios.

\section{Discussion}

For practical applications, the amount of crosstalk, i.e., the ratio of the remaining original signal power at the location of an idler or vice versa, must remain as low as possible. With this technique, it is entirely determined by the quality of the interleaver, and with current commercial devices, a crosstalk level that is better than $-40 \mathrm{~dB}$ can in principle be obtained. Interleaving filters should also introduce negligible dispersion for the proposed configuration to be cascadable. Dispersionfree interleavers have already been proposed [22].

It should be noted that the number of spurious FWM components in an OPA [23], [24] grows approximately like the cube of the total number of WDM channels. Therefore, by using half of the channels in each branch, the number of FWM components is reduced by about a factor of 8 . This is the secondary benefit of partitioning the input signals.

Ideally, the two OPAs in either branch should be identical. However, the HNL-DSFs used in practice have dispersion characteristics that vary with distance and usually different average zero-dispersion wavelengths [8], [25]. Therefore, they will exhibit different gain spectra when driven by the same pump wavelengths. This can be partly compensated by using different pump powers in each branch and possibly by using slightly different pump wavelengths, which were optimized to match the gain profiles of the two OPAs.

Last, the noise performance of the proposed configurations needs to be thoroughly investigated. Our measurements were performed with moderate gains, and therefore, possible effects of OPA noise might not have been revealed. On the same issue, it should be noted that the interleaver suppresses the idlers superimposed on the signals but not the noise transferred from the idler band to the signal band during amplification. This effect might cause additional penalty at higher OPA gains.

\section{CONCLUSiOn}

We have introduced a method that permits the utilization of the full bandwidth of fiber OPAs when used with a full-WDM spectrum. We have investigated the filter characteristics required for the implementation of the proposed architectures. We have discussed the tradeoffs involved in the use of interleavers and high-pass band filters. We have performed experiments with a four-signal spectrum and demonstrated the ability to separate the idlers from the amplified signal spectrum, with a crosstalk of below $-20 \mathrm{~dB}$ and subdecibel power penalty. This method can help make fiber OPAs practical for applications in future wideband DWDM systems either amplifiers or wavelength converters. Most aspects of the method introduced here are also applicable to other types of devices, including 
wavelength converters based on cascaded $\chi^{(2)}$ nonlinear effects [26].

\section{ACKNOWLEDGMENT}

The authors would like to thank Dr. M. Onishi of Sumitomo Electric Industries, Ltd., for providing the HNL-DSF, OIDA for providing the high-power EDFA and filters, and Sprint Advanced Technology Laboratories for the loan of equipment.

\section{REFERENCES}

[1] M. E. Marhic, K. K.-Y. Wong, G. Kalogerakis, and L. G. Kazovsky, "Toward practical fiber optical parametric amplifiers and oscillators," Optics Photonics News, vol. 15, no. 9, pp. 22-27, Sep. 2004.

[2] M. Ho, K. Uesaka, M. E. Marhic, Y. Akasaka, and L. G. Kazovsky, "200-nm-bandwidth fiber optical amplifier combining parametric and Raman gain," J. Lightw. Technol., vol. 19, no. 7, pp. 977-981, Jul. 2001.

[3] J. Hansryd and P. A. Andrekson, "Broad-band continuous-wave-pumped fiber optical parametric amplifier with $49-\mathrm{dB}$ gain and wavelengthconversion efficiency," IEEE Photon. Technol. Lett., vol. 13, no. 3, pp. 194-196, Mar. 2001

[4] M. E. Marhic, N. Kagi, T. K. Chiang, and L. G. Kazovsky, "Broadband fiber optical parametric amplifiers," Opt. Lett., vol. 21, no. 8, pp. 573-575, Apr. 1996

[5] M. E. Marhic, K. K.-Y. Wong, and L. G. Kazovsky, "Wideband tuning of the gain spectra of one-pump fiber optical parametric amplifiers," IEEE J. Sel. Topics Quantum Electron., vol. 10, no. 5, pp. 1133-1141, Sep./Oct. 2004.

[6] K. K.-Y. Wong, M. E. Marhic, G. Kalogerakis, and L. G. Kazovsky, "Fiber optical parametric amplifier and wavelength converter with record $360 \mathrm{~nm}$ gain bandwidth and $50 \mathrm{~dB}$ signal gain," presented at the Conf. Lasers and Electro-Optics, Baltimore, MD, Jun. 2003, Paper CThPDB6.

[7] K. K.-Y. Wong, K. Shimizu, K. Uesaka, G. Kalogerakis, M. E. Marhic, and L. G. Kazovsky, "Continuous-wave fiber optical parametric amplifier with $60 \mathrm{~dB}$ gain using a novel two-segment design," IEEE Photon. Technol. Lett., vol. 15 , no. 12 , pp. 1707-1709, Dec. 2003.

[8] M. Hirano, T. Nakanishi, T. Okuno, and M. Onishi, "Broadband wavelength conversion over 193-nm by HNL-DSF improving higher-order dispersion performance," in Proc. ECOC, Glasgow, U.K., Sep. 2005, vol. 6, pp. 43-46.

[9] J. H. Lee, T. Nagashima, T. Hasegawa, S. Ohara, N. Sugitomo, T. Tanemura, and K. Kikuchi, "Wavelength conversion of 40-Gb/s NRZ signal using four-wave-mixing in 40-cm-long Bismuth Oxide based highly-nonlinear optical fiber," presented at the Optical Fiber Commun. Conf. (OFC), Anaheim, CA, Mar. 2005, DP23.

[10] M. E. Marhic, Y. Park, F. S. Yang, and L. G. Kazovsky, "Broadband fiber optical parametric amplifiers," Opt. Lett., vol. 21, no. 17, pp. 1354-1356, Sep. 1996.

[11] M. N. Islam and O. Boyraz, "Fiber parametric amplifiers for wavelength band conversion," IEEE J. Sel. Topics Quantum Electron., vol. 8, no. 3, pp. 527-537, May-Jun. 2002.

[12] S. Haykin, Communication Systems, 3rd ed. New York: Wiley, 1994.

[13] R. A. Spanke, "Architectures for large nonblocking optical space switches," IEEE J. Quantum Electron., vol. QE-22, no. 6, pp. 964-967, Jun. 1986.

[14] M. E. Marhic, G. Kalogerakis, and L. G. Kazovsky, "Gain reciprocity in fibre optical parametric amplifiers," Electron. Lett., vol. 42, no. 9, pp. 519520, Apr. 2006.

[15] K. Jinguji and M. Kawachi, "Synthesis of coherent two-port lattice-form optical delay-line circuit," J. Lightw. Technol., vol. 13, no. 1, pp. 73-82, Jan. 1995.

[16] M. E. Marhic, G. Kalogerakis, K. Uesaka, K. Shimizu, K. K.-Y. Wong, and L. G. Kazovsky, "Interleaver-based method for full utilization of the bandwidth of fiber optical parametric amplifiers and wavelength converters," presented at the Optical Fiber Commun. Conf. (OFC), Atlanta, GA, Mar. 2003, ThK4

[17] S.-K. Liaw, K.-P. Ho, C. Lin, and S. Chi, "Multichannel bidirectional transmission using a WDM MUX/DMUX pair and unidirectional in-line amplifiers," IEEE Photon. Technol. Lett., vol. 9, no. 12, pp. 1664-1666, Dec. 1997.

[18] J. L. Blows and S. E. French, "Low-noise-figure optical parametric amplifier with a continuous-wave frequency-modulated pump," Opt. Lett., vol. 27, no. 7, pp. 491-493, Apr. 2002.
[19] A. Durecu-Legrand, D. Bayart, A. Mussot, T. Sylvestre, E. Lantz, and H. Maillotte, "Impact of pump OSNR on noise figure for fiber-optical parametric amplifiers," IEEE Photon. Technol. Lett., vol. 17, no. 6, pp. 1178-1180, Jun. 2005.

[20] M. E. Marhic, G. Kalogerakis, K. K.-Y. Wong, and L. G. Kazovsky, "Pump to signal transfer of low-frequency intensity modulation in fiber optical parametric amplifiers," J. Lightw. Technol., vol. 23, no. 3, pp. 1049-1055, Mar. 2005.

[21] T. Torounidis, H. Sunnerud, O. Hedekvist, and A. Andrekson, "Amplification of WDM signals in fiber-based optical parametric amplifiers," IEEE Photon. Technol. Lett., vol. 15, no. 8, pp. 1061-1063, Aug. 2003.

[22] T. Chiba, H. Arai, K. Oshira, H. Nonen, H. Okano, and H. Uetsuka, "Novel architecture of wavelength interleaving filter with Fourier transform-based MZIs," presented at the Optical Fiber Commun. Conf. (OFC), Murray Hill, NJ, 2000, Paper WB5-1.

[23] J. L. Blows and P.-F. Hu, "Cross-talk-induced limitations of two-pump optical fiber parametric amplifiers," J. Opt. Soc. Amer. B, Opt. Phys., vol. 21, no. 5, pp. 989-995, May 2004.

[24] F. A. Callegari, J. M. Chavez Boggio, and H. L. Fragnito, "Spurious fourwave mixing in two-pump fiber-optic parametric amplifier," IEEE Photon. Technol. Lett., vol. 16, no. 2, pp. 434-436, Feb. 2004.

[25] A. Mussot, E. Lanz, T. Sylvestre, H. Maillotte, A. Durecu-Legrand, C. Simonneau, and D. Bayart, "Zero-dispersion wavelength mapping of a highly nonlinear optical fibre-based parametric amplifier," presented at the Eur. Conf. Optical Commun. (ECOC), Stockholm, Sweden, Sep. 2004, Tu3.3.7.

[26] M. H. Chou, I. Brenner, M. M. Fejer, E. E. Chaban, and S. B. Christman, "1.5- $\mu \mathrm{m}$-band wavelength conversion based on cascaded second-order nonlinearity in $\mathrm{LiNbO}_{3}$ waveguides," IEEE Photon. Technol. Lett., vol. 11, no. 6, pp. 653-655, Jun. 1999.

Georgios Kalogerakis (S'02), photograph and biography not available at time of publication.

Michel E. Marhic (SM'89), photograph and biography not available at time of publication.

Katsumi Uesaka (M'01), photograph and biography not available at time of publication.

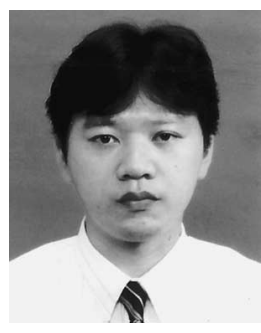

Katsuhiro Shimizu (M'93) received the B.E. and M.E. degrees in electrical engineering from the University of Tokyo, Tokyo, Japan, in 1989 and 1991, respectively.

Since 1991, he has been with the Information Technology R\&D Center, Mitsubishi Electric Corporation, Itami, Japan, working on research and development on optical wavelength-division-multiplexing technologies and long-haul transmission systems that employ optical amplifiers.

Mr. Shimizu is a member of the IEEE Communications Society, the IEEE Lasers and Electro-Optics Society, the IEEE Oceanic Engineering Society, the Optical Society of America, and the Institute of Electronics, Information, and Communication Engineers of Japan.

Kenneth Kin-Yip Wong (S'00), photograph and biography not available at time of publication.

Leonid G. Kazovsky (M'80-SM'83-F'91), photograph and biography not available at time of publication. 